\title{
The Research Progress of Electrophysiological Technology in Premature Ovarian Insufficiency
}

\author{
Afsarunnesa Syeda ${ }^{1}$, Li Fan ${ }^{1 *}$ \\ ${ }^{1}$ Department of Obstetrics and Gynaecology, Renmin Hospital, Hubei University of \\ Medicine, Shiyan, Hubei, China \\ *Corresponding to: Prof. Dr. Li Fan; Department of Obstetrics and Gynaecology, Renmin \\ Hospital, Hubei University of Medicine, Shiyan, Hubei, 442000, China; Email: \\ 49555709@qq.com
}

\begin{abstract}
Ovarian hypofunction or primary ovarian insufficiency (POI) is a common cause in the etiology of female infertility. It is a heterogeneous disorder, affecting approximately $1 \%$ of women before the age of 40 . The characteristics of POI are menstrual disorders, including amenorrhea and delayed menstruation, accompanied by a raised gonadotrophin level and decreased estradiol level. In recent years, electrophysiology (EP) technology has been advancing rapidly in the diagnosis and treatments of numerous diseases. According to studies, bioelectrical stimulation (BES) therapy is an electrophysiology technology that plays significant roles in numerous diseases, including on the thin endometrium in patients with POI. In this review, the overall research progress of this electrophysiological technology has been discussed in relation to the advancement of primary ovarian insufficiency.
\end{abstract}

Keywords: Primary ovarian insufficiency; ovarian hypofunction; amenorrhea; follicular stimulating hormone; electrophysiological technology; bioelectrical stimulation

\section{Introduction}

Ovarian hypofunction or premature ovarian insufficiency is a subclass of ovarian dysfunction in which the cause is within the ovary. In most cases, an unknown mechanism leads to premature exhaustion of the resting pool of primordial follicles. Primary ovarian insufficiency might also result from genetic defects, chemotherapy, radiotherapy, or surgery [1]. Primary ovarian insufficiency occurs through two major mechanisms: follicle dysfunction and follicle depletion ${ }^{[2]}$. In POI, ovarian follicles decrease below a certain threshold and lose the potential for ovulation, leading to amenorrhea ${ }^{[3]}$. The incidence of POI has been reported 
to be $1 \%$ in women younger than 40 and $0.1 \%$ among women under $30^{[1-3]}$. It has widespread physical and psychological consequences due to the symptoms and long-term effects of sex steroid deficiency ${ }^{[4]}$. In most of the women affected, there are no signs or symptoms that precede the interruption of menstruation and the onset of POI. The majority of women have a typical history of menarche, regular menstrual cycles, and normal fertility ${ }^{[5]}$. The number of reproductive years among women varies depending on steroid production by the ovaries. There is no consensus on criteria to identify primary ovarian insufficiency in adolescents, and delay in diagnosis is typical ${ }^{[6]}$. Proper diagnostic criteria for POI are currently lacking. However, oligomenorrhea or amenorrhea for at least 4 months and persistently elevated folliclestimulating hormone (FSH) levels that exceed 25-40 IU/L on two occasions more than 4 weeks apart in women under the age of 40 defines POI clinically ${ }^{[3,7]}$. Initial laboratory evaluation for suspected primary ovarian insufficiency includes measurements of basal FSH and basal estradiol levels and tests to rule out causes such as pregnancy, thyroid disease, and hyperprolactinemia ${ }^{[7]}$. 5-10\% of women with POI experience spontaneous conception and delivery, where a previous study indicated that the incidence of low birth weight (LBW) among mothers having normal vaginal delivery was almost $8.87 \%$. This indicates that children born of POI patients also have other complications, including LBW ${ }^{[8]}$. Thus, primary ovarian insufficiency can be distinguished from natural menopause and may be described as a decreased ovarian reserve ${ }^{[2,9]}$.

In about $90 \%$ of cases, the exact cause of POI is unknown. In cases of primary ovarian insufficiency that are not associated with a syndrome, the laboratory tests recommended determining the cause include a karyotype analysis and testing for a fragile $\mathrm{X}$ mental retardation 1 (FMR1) premutation ${ }^{[2-3,10-12]}$. Pelvic ultrasonography should also be performed. The diagnosis of primary ovarian insufficiency affects a woman's physical and emotional wellbeing, and the management of the condition should address both ${ }^{[2]}$. Studies recommended estrogen-replacement therapy for women with primary ovarian insufficiency ${ }^{[3,13]}$. A recent study found that thin endometrium incidence in patients with premature ovarian insufficiency ${ }^{[14]}$, characterized by the endometrial thickness, is less than or equal to $7 \mathrm{~mm}^{[15]}$. A recent study also finds that electrophysiological technology, such as bioelectrical stimulation on the pelvic floor in patients with premature ovarian insufficiency affects endometrial thickness ${ }^{[16]}$. In this review, the research progress on premature ovarian insufficiency in relation to electrophysiology technology has been conducted. 


\section{An Overview of Premature Ovarian Insufficiency}

Premature ovarian insufficiency represents a condition characterized by the absence of normal ovarian function due to an incipient by 3 to 10 years ovarian aging ${ }^{[5]}$. The first case of premature ovarian insufficiency was reported in 1942 by Fuller Albright in young women characterized by menopausal FSH levels and low estrogen levels amenorrhea. This condition has been heterogeneously termed "premature ovarian insufficiency." Later it was recognized under several other names, such as the preferred term "premature ovarian failure" or "premature menopause" [17-19]. Other terms used for this condition include primary ovarian failure and hypergonadotropic hypogonadism, as well as the misnomer, gonadal dysgenesis ${ }^{[2}$, ${ }^{20]}$. The prevalence is $1: 10,000$ at the age of $18-25,1: 1000$ in women aged 25-30 years, and 1:100 in the age range of 35-40 years. The incidence of POI may vary depending on ethnic people, and it has a familiar occurrence in about $15 \%$ of total incidence ${ }^{[19-23]}$. In women with primary ovarian insufficiency, systemic hormone replacement therapy (HRT) is a practical approach to treat the symptoms of hypoestrogenism and mitigate long-term health risks if there are no contraindications to treatment. HRT is indicated to reduce the risk of osteoporosis, cardiovascular disease, and urogenital atrophy and to improve the quality of life of women with primary ovarian insufficiency ${ }^{[24]}$.

\section{Therapeutic Potential of Electrophysiological (EP) Technology in Premature Ovarian Insufficiency}

Electrophysiology technology has been advancing rapidly in the past few years with new ablation tools in the treatments of numerous diseases, miniaturized diagnostic monitoring systems, and new implantable rhythm management devices that make procedures much less invasive. Electrophysiological technology has become a beneficial diagnostic tool such as electrocardiograms (ECG), electromyograms (EMG), electroencephalography (EEG) and treatment methods in medical science, such as shock defibrillation and radiofrequency techniques, pacemakers. ${ }^{[25-31]}$. Cardiac electrophysiology has evolved into an important subspecialty in cardiovascular medicine due to the significant advances in the treatment of heart rhythm disorders ${ }^{[32]}$. The rapid development of cellular electrophysiology, molecular biology, genetics, computer modeling, and imaging has led to the exponential growth of knowledge in basic cardiac electrophysiology. Magnetic resonance-guidance of electrophysiological procedures also offer the potential for enhanced arrhythmia substrate assessment, improved procedural guidance, and real-time assessment of ablation lesion formation ${ }^{[32-33]}$. Multiple studies have been done in recent years that explain the therapeutic 
potentials of electrophysiology technology, such as bioelectrical stimulation therapy in various diseases. These studies including in arthritis ${ }^{[34]}$, asthma ${ }^{[35]}$, diabetes ${ }^{[36]}$, digestive disorders [37], Alzheimer's disease ${ }^{[38]}$, depression ${ }^{[39]}$, cardiovascular disease ${ }^{[40]}$, cognition ${ }^{[41]}$. Several studies also reported that this electrophysiological technology could be beneficial in the diagnosis and treatment of obstetrics ${ }^{[29]}$ and gynecological diseases ${ }^{[30,32,42]}$, including in the premature ovarian insufficiency. A recent study also indicated that there had been a significant effect of bioelectrical stimulation is done on the pelvic floor on thin endometrium ${ }^{[17]}$. Thus, these reports indicate that further investigations are required using electrophysiology technology-based intervention on patients with premature ovarian insufficiency to see if there are any changes in the endometrial thickness, which may reach more susceptibility of conception.

\section{Conclusion and Expectation}

The electrophysiological technology, including bioelectrical stimulation therapy, is a convergence of advances in medical science. The therapeutic potentials of electrophysiological technology are an added advantage in numerous diseases along with its pre-existing medical or surgical therapies. To be more specific, the thin endometrium in patients with premature ovarian insufficiency is a common cause of the failure of embryo implantation. In relation to traditional hormone replacement therapy, bioelectrical stimulation therapy is a safe, drug-free treatment option for these diseases. Thus, further investigation on thin endometrium-based patients with premature ovarian insufficiency may get benefits for conception.

\section{Acknowledgments}

Author thanks the Renmin Hospital, Hubei University of Medicine, for all the supports.

\section{Conflict of Interests}

None.

\section{REFERENCES}

[1] De Vos M, Devroey P, Fauser BC. Primary ovarian insufficiency [J]. The Lancet, 2010, 376(9744):911-921.

[2] Nelson LM. Primary Ovarian Insufficiency [J]. New England Journal of Medicine, 2009, 360(6):606-614. 
[3] Lee HN, Chang EM. Primordial follicle activation as new treatment for primary ovarian insufficiency [J]. Clinical and Experimental Reproductive Medicine, 2019, 46(2):43-49.

[4] Maclaran K, Panay N. Current Concepts in Premature Ovarian Insufficiency [J]. Women's Health, 2015, 11(2):169-182.

[5] Luisi S, Orlandini C, Regini C, et al. Premature ovarian insufficiency: from pathogenesis to clinical management [J]. Journal of Endocrinological Investigation, 2015, 38(6):597603.

[6] American College of Obstetricians and Gynecologists. Committee Opinion No. 605 [J]. Obstetrics \& Gynecology, 2014, 124(1): 193-197.

[7] Webber L, Davies M, Anderson R, et al. ESHRE Guideline: management of women with premature ovarian insufficiency [J]. Human Reproduction, 2016, 31(5):926-937.

[8] Asaduzzaman A, Karim MR, Amin A, et al. The relationship of birth weight with occipitofrontal circumference and mid upper arm circumference: an alternative means to detect LBW [J]. International Journal of Dental and Health Sciences, 2017, 4(5):10991113.

[9] Practice Committee of the American Society for Reproductive Medicine. Testing and interpreting measures of ovarian reserve: a committee opinion $[\mathrm{J}]$. Fertility and Sterility, 2012, 98(6):1407-1415.

[10] McConkie-Rosell A, Finucane B, Cronister A, et al. Genetic Counseling for Fragile X Syndrome: Updated Recommendations of the National Society of Genetic Counselors [J]. Journal of Genetic Counseling, 20015, 14(4):249-270.

[11] Sherman S, Pletcher BA, Driscoll DA. Fragile X syndrome: Diagnostic and carrier testing [J]. Genetics in Medicine, 2005, 7(8), 584-587.

[12] American College of Obstetricians and Gynecologists. ACOG Committee Opinion No. 338: Screening for Fragile X Syndrome [J]. Obstetrics \& Gynecology, 2006, 107(6):1483.

[13] Pines A, Sturdee DW, Birkhäuser MH, et al. IMS Updated Recommendations on postmenopausal hormone therapy [J]. Climacteric, 2007, 10(3):181-194.

[14] Kasius A, Smit JG, Torrance HL, et al. Endometrial thickness and pregnancy rates after IVF: a systematic review and meta-analysis [J]. Human Reproduction Update, 2014, 20(4):530-541.

[15] Mahajan N, Sharma S. The endometrium in assisted reproductive technology: How thin is thin [J]? Journal of Human Reproductive Sciences, 2016, 9(1):3. 
[16] Yuan B, Zheng H, Xu M, et al. Effects of pelvic floor bioelectrical stimulation on frozen embryo transfer patients with thin endometrium [J]. European Journal of Inflammation, 2019, 17:205873921882286.

[17] Albright F, Smith PH, Fraser R. A syndrome characterized by primary ovarian insufficiency and decreased stature [J]. The American Journal of the Medical Sciences, 1942, 204(5):625-648.

[18] Hubayter ZR, Popat V, Vanderhoof VH, et al. A prospective evaluation of antral follicle function in women with 46,XX spontaneous primary ovarian insufficiency [J]. Fertility and Sterility, 2010, 94(5):1769-1774.

[19] Rudnicka E, Kruszewska J, Klicka K, et al. Premature ovarian insufficiency aetiopathology, epidemiology, and diagnostic evaluation [J]. Menopausal Review, 2018, 17(3):105-108.

[20] Welt CK. Primary ovarian insufficiency: a more accurate term for premature ovarian failure [J]. Clinical Endocrinology, 2018, 68(4):499-509.

[21] Franić D. Genetic etiology of primary premature ovarian insufficiency [J]. Acta Clinica Croatia, 2016:629-635.

[22] Cramer DW, Xu H. Predicting age at menopause [J]. Maturitas, 1996, 23(3):319-326.

[23] Luborsky J, Meyer P, Sowers M, et al. Premature menopause in a multi-ethnic population study of the menopause transition [J]. Human Reproduction, 2003, 18(1):199206.

[24] American College of Obstetricians and Gynecologists. Committee Opinion No. 698 [J]. Obstetrics \& Gynecology, 2017, 129(5):e134-e141.

[25] Shabeeb D, Najafi M, Hasanzadeh G, et al. Electrophysiological measurements of diabetic peripheral neuropathy: A systematic review [J]. Diabetes \& Metabolic Syndrome: Clinical Research \& Reviews, 2018, 12(4):591-600.

[26] Mahajan R, Lau DH, Brooks AG, et al. Electrophysiological, Electroanatomical, and Structural Remodeling of the Atria as Consequences of Sustained Obesity [J]. American College of Cardiology, 2015, 66(1):1-11.

[27] Lee SA, Miller JF, Watrous AJ, et al. Electrophysiological Signatures of Spatial Boundaries in the Human Subiculum [J]. Neuroscience, 2018, 38(13):3265-3272.

[28] István P, Szilágyi A, Kellényi L. Electrophysiology in obstetrics [J]. European Journal of Obstetrics \& Gynecology and Reproductive Biology, 2016, 206:e91.

[29] Cappellano F. Electrical Stimulation for Pelvic Pain [J]. Electrical Stimulation for Pelvic Floor Disorders, 2014:225-233. 
[30] Martellucci J. Electrical Stimulation in Sexual Dysfunction [J]. Electrical Stimulation for Pelvic Floor Disorders, 2014:201-223.

[31] Syeda A, Karim MR. The mean age of petit mal epilepsy [J]. Journal of Pediatric Neurosciences, 2016, 11(2):112-114.

[32] Lau DH, Volders PG, Kohl P, et al. Opportunities and challenges of current electrophysiology research: a plea to establish 'translational electrophysiology' curricula [J]. Europace, 2015, 17(5):825-833.

[33] Chubb H, Williams SE, Whitaker J, et al. Cardiac Electrophysiology Under MRI Guidance: an Emerging Technology [J]. Arrhythmia \& Electrophysiology Review, 2015, $6(2): 85$.

[34] Genovese MC, Gaylis N, Sikes D, et al. LB009 First-in-human study of novel implanted vagus nerve stimulation device to treat rheumatoid arthritis [J]. Annals of the Rheumatic Diseases, 2019, 78:264.

[35] Staats P, Emala C, Simon B, et al. Neurostimulation for Asthma [J]. Neuromodulation, 2018:1339-1345.

[36] Masi EB, Levy T, Tsaava T, et al. Identification of hypoglycemia-specific neural signals by decoding murine vagus nerve activity [J]. Bioelectronic Medicine, 2019, 5(1).

[37] Payne SC, Furness JB, Burns O, et al. Anti-inflammatory Effects of Abdominal Vagus Nerve Stimulation on Experimental Intestinal Inflammation [J]. Frontiers in Neuroscience, 2019, 13.

[38] Kaczmarczyk R, Tejera D, Simon BJ, et al. Microglia modulation through external vagus nerve stimulation in a murine model of Alzheimer's disease [J]. Journal of Neurochemistry, 2017, 146:76-85.

[39] Sackeim H. Vagus Nerve Stimulation (VNS ${ }^{\mathrm{TM}}$ ) for Treatment-Resistant Depression Efficacy, Side Effects, and Predictors of Outcome [J]. Neuropsychopharmacology, 2001, 25(5):713-728.

[40] Zhou L, Filiberti A, Humphrey MB, et al. Low-level transcutaneous vagus nerve stimulation attenuates cardiac remodelling in a rat model of heart failure with preserved ejection fraction [J]. Experimental Physiology, 2018, 104(1):28-38.

[41] Sanders TH, Weiss J, Hogewood L, et al. Cognition-Enhancing Vagus Nerve Stimulation Alters the Epigenetic Landscape [J]. The Journal of Neuroscience, 2019:2407-2418.

[42] Grifantini K. Electrical Stimulation: A Panacea for Disease? DARPA Investigates New Bioelectrical Interfaces for a Range of Disorders [J]. IEEE Pulse, 2016, 7(4):30-35. 